\title{
Differential Immunohistochemical Profiles for Distinguishing Prostate Carcinoma and Urothelial Carcinoma
}

\author{
Woo Jin $\mathrm{Oh}^{*}$ · Arthur Minwoo Chung* \\ Jee Soon Kim · Ji Heun Han \\ Sung Hoo Hong ${ }^{1} \cdot$ Ji Yeol Lee \\ Yeong Jin Choi \\ Departments of Hospital Pathology and ${ }^{1}$ Urology, \\ Seoul St. Mary's Hospital, College of Medicine, \\ The Catholic University of Korea, Seoul, Korea \\ Received: February 20, 2016 \\ Revised: May 21, 2016 \\ Accepted: June 14, 2016 \\ Corresponding Author \\ Yeong Jin Choi, MD, PhD \\ Department of Hospital Pathology, \\ Seoul St. Mary's Hospital, College of Medicine, \\ The Catholic University of Korea, 222 Banpo-daero, \\ Seocho-gu, Seoul 06591, Korea \\ Tel: +82-2-2258-1616 \\ Fax: $+82-2-2258-1627$ \\ E-mail: mdyjchoi@catholic.ac.kr \\ *Woo Jin Oh and Arthur Minwoo Chung contributed \\ equally to this work.
}

Background: The pathologic distinction between high-grade prostate adenocarcinoma (PAC) involving the urinary bladder and high-grade urothelial carcinoma (UC) infiltrating the prostate can be difficult. However, making this distinction is clinically important because of the different treatment modalities for these two entities. Methods: A total of 249 patient cases (PAC, 111 cases; UC, 138 cases) collected between June 1995 and July 2009 at Seoul St. Mary's Hospital were studied. An immunohistochemical evaluation of prostatic markers (prostate-specific antigen [PSA], prostate-specific membrane antigen [PSMA], prostate acid phosphatase [PAP], P501s, NKX3.1, and $\alpha$-methylacyl coenzyme A racemase [AMACR]) and urothelial markers (CK34ßE12, p63, thrombomodulin, S100P, and GATA binding protein 3 [GATA3]) was performed using tissue microarrays from each tumor. Results: The sensitivities of prostatic markers in PAC were 100\% for PSA, 83.8\% for PSMA, 91.9\% for PAP, 93.7\% for P501s, 88.3\% for NKX 3.1, and 66.7\% for AMACR. However, the urothelial markers CK34ßE12, p63, thrombomodulin, S100P, and GATA3 were also positive in $1.8 \%, 0 \%, 0 \%, 3.6 \%$, and $0 \%$ of PAC, respectively. The sensitivities of urothelial markers in UC were $75.4 \%$ for CK34ßE12, 73.9\% for p63, 45.7\% for thrombomodulin, $22.5 \%$ for S100P, and $84.8 \%$ for GATA3. Conversely, the prostatic markers PSA, PSMA, PAP, P501s, NKX3.1, and AMACR were also positive in $9.4 \%, 0.7 \%, 18.8 \%, 0.7 \%, 0 \%$, and $8.7 \%$ of UCs, respectively. Conclusions: Prostatic and urothelial markers, including PSA, NKX3.1, p63, thrombomodulin, and GATA3 are very useful for differentiating PAC from UC. The optimal combination of prostatic and urothelial markers could improve the ability to differentiate PAC from UC pathologically.

Key Words: Prostatic adenocarcinoma; Urinary bladder; Immunohistochemistry; Pathologic diagnosis; Urothelial carcinoma
Prostate involvement by urothelial carcinoma (UC) can occur from direct invasion of an infiltrating UC into the prostate stroma and from intraductal extension of the UC with or without subsequent stromal invasion of the prostate. ${ }^{1}$ The involvement of the urinary bladder by prostate adenocarcinoma (PAC) as a metastasis or by direct extension occurs in $12 \%$ of all secondary bladder tumors ${ }^{1}$ and is the second most common origin of this bladder cancer. ${ }^{2}$ A common diagnostic problem in this circumstance is to differentiate between high-grade UC and high-grade PAC. Distinguishing between these two entities is crucial because the treatment for PAC is very different from that of infiltrating UC. Advanced UC is generally managed with chemotherapy, whereas advanced PAC is often managed with anti-androgen hormone therapy.

In the absence of a papillary UC or noninvasive flat component, distinguishing high-grade PAC involving the bladder from infiltrating UC on routine hematoxylin and eosin stain can be challenging. ${ }^{3}$ Even in cases in which a known history of PAC is available, superimposed histologic changes, such as squamous metaplasia caused by prior radiation or hormonal therapy or poor differentiation, lead to difficulty in differentiating a primary UC from a recurrent PAC on a needle biopsy or transurethral resection of prostate. ${ }^{2}$ Given the equivocal histologic features and significant differences in treatment modality and prognosis, immunohistochemistry is necessary whenever the differential diagnosis cannot be made with complete certainty based on histologic features only.

Prostate-specific antigen (PSA) and prostate acid phosphatase (PAP) have been known to assist in verifying the prostatic lineage in cases of metastatic carcinoma of unknown origin. ${ }^{4}$ However, in poorly differentiated carcinomas, the sensitivities of PSA and PAP decrease. ${ }^{3}$ Newer prostatic markers, such as prostein (P501s), prostate-specific membrane antigen (PSMA), proPSA, and NKX3.1, may provide added utility. ${ }^{3}$ Urothelial markers, such 
as CK34ßE12, p63, thrombomoculin, S100P, and GATA binding protein 3 (GATA3), have been reported to be useful in identifying tumors of urothelial origin. However, thrombomodulin is only moderately sensitive compared with CK34ßE12 and p63 in identifying UC., ${ }^{2,3}$ Cytokeratin 7 and cytokeratin 20 are of limited utility in the differential diagnosis of these tumors because they may both be positive in a subset of PAC and UC. ${ }^{5-7}$

In the present study, we analyzed and evaluated the diagnostic utility of prostatic and urothelial immunohistochemical markers in PAC and UC with variable differentiation.

\section{MATERIALS AND METHODS}

\section{Patients and materials}

We performed a retrospective analysis of a prospectively maintained database of patients approved by the Institutional Review Board of Seoul St. Mary's Hospital, the Catholic University of Korea (KC13SISI0909). We enrolled a total of 111 patients with PAC and 138 patients with UC who were treated at Seoul St. Mary's Hospital between June 1995 and July 2009.

Cases of PAC were divided according to low (Gleason score $<7$ ) and high grade (Gleason score 27 ). Of the 111 PACs from radical prostatectomy specimens, 64 cases $(57.6 \%)$ were low grade and $47(42.3 \%)$ were high grade. Cases of UC, whether noninvasive papillary or infiltrating, were divided into low and high grade according to the World Health Organization classification. Of the 138 UCs from cystectomy or tranurethral resection of bladder specimens, 28 cases (20.3\%) were noninvasive papillary low grade, and $110(79.7 \%)$ were noninvasive papillary or infiltrating cases with high-grade morphology. The male: female ratio was 7:1. None of the patients received neoadjuvant chemotherapy, hormone, or radiation therapy.

\section{Immunohistochemistry}

We analyzed and evaluated the immunoprofile of urothelial and prostatic markers using tissue microarrays (TMAs). Needle punches (0.5-mm diameter) of paraffin-embedded tissue blocks were transferred and arrayed in the recipient block. Tissue punches yielding equivocal results were excluded from data analysis. Only interpretable positive or negative staining was accepted as a positive or negative result. Five-micrometer sections from the TMA blocks were prepared for immunohistochemical staining. All tissues were fixed in neutral buffered formalin, paraffin embedded, and processed in a standard tissue processor.

The immunohistochemical stains were performed on an automated immunostainer (LV-1 Autostainer, Lab Vision, Fremont,
CA, USA) using the standard avidin-biotin peroxidase method after antigen retrieval according to the manufacturer's instructions. The immunohistochemical stains for this study included PSA, PSMA, PAP, P501s, NKX3.1, $\alpha$-methylacyl coenzyme A racemase (AMACR), CK34ßE12, p63, thrombomodulin, S100P, and GATA3. Primary antibodies used in this study were as follows: PSA (polyclonal, Dako, Carpinteria, CA, USA), PSMA (monoclonal, Novocastra Lab, Ltd., Newcastle upon Tyne, UK), PAP (monoclonal, Dako), P501s (monoclonal, Dako), NKX3.1 (monoclonal, Athena ES, Baltimore, MD, USA), AMACR (monoclonal, Cell Marque, Rocklin, CA, USA), CK34ßE12 (monoclonal, Dako), p63 (monoclonal, Lab Vision), thrombomodulin (monoclonal, Dako), S100P (monoclonal, Dako), and GATA3 (monoclonal, prediluted, Cell Marque). Detailed information about the antibodies used in this study is included in Table 1. The staining patterns for PSA, PSMA, AMACR, and thrombomodulin were cytoplasmic. The P501s staining was in a perinuclear cytoplasmic location and had a speckled pattern. CK34ßE12 showed membranous and/or cytoplasmic staining. Only nuclear stains with or without cytoplasmic staining were accepted as positive results for the p63, NKX3.1, S100P, and GATA3 stains. All immunohistochemical staining reactions were reviewed by experienced genitourinary pathologists. The patterns of all immunostaining markers in this study were diffuse and homogeneous. The immunohistochemical staining results were recorded as positive stains when $\geq 10 \%$ of cells showed positive reaction regardless of the intensity of the staining. SPSS ver. 16.0 (SPSS Inc., Chicago, IL, USA) was used for statistic analysis. We used chi-square tests to analyse the reactivity of all antibody panels. p-values $\leq .05$ were considered statistically significant.

Table 1. Antibodies used in immunohistochemical staining

\begin{tabular}{llll}
\hline Antibody & Clone & Dilution & Vendor \\
\hline PSA & Polyclonal & $1: 1,000$ & Dako \\
PSMA & Monoclonal & $1: 100$ & Novocastra \\
PAP & Monoclonal & Prediluted & Dako \\
P501s & Monoclonal & $1: 200$ & Dako \\
NKX3.1 & Monoclonal & $1: 500$ & Athena ES \\
AMACR & Monoclonal & $1: 200$ & Cell Marque \\
CK34ßE12 & Monoclonal & $1: 50$ & Dako \\
p63 & Monoclonal & $1: 800$ & Lab Vision \\
TM & Monoclonal & $1: 1,000$ & Dako \\
S100P & Monoclonal & $1: 800$ & Dako \\
GATA3 & Monoclonal & Prediluted & Cell Marque
\end{tabular}

PSA, prostate-specific antigen; PSMA, prostate-specific membrane antigen; PAP, prostate acid phosphatase; AMACR, $\alpha$-methylacyl coenzyme A racemase; TM, thrombomodulin; GATA3, GATA binding protein 3. 


\section{RESULTS}

The results for PSA, PSMA, PAP, P501s, NKX3.1, AMACR, CK34ßE12, p63, thrombomodulin, S100P, and GATA3 were summarized (Table 2).

\section{Prostatic markers in the PAC group}

The sensitivities of prostatic markers in PAC were as follows: PSA (100\%), PSMA (83.8\%), PAP (91.9\%), P501s (93.7\%), NKX3.1 (88.3\%), and AMACR (66.7\%). The specificities of prostatic markers in PAC were as follows: PSA (90.6\%), PSMA (99.3\%), PAP (81.2\%), P501s (99.3\%), NKX3.1 (100\%), and AMACR (91.3\%). The positive predictive values (PPVs) of prostatic markers in PAC were as follows: PSA (89.5\%), PSMA (98.9\%), PAP (79.7\%), P501s (99.1\%), NKX3.1 (100\%), and AMACR (86.1\%). The negative predictive values (NPVs) of prostatic markers in PAC were as follows: PSA (100\%), PSMA (88.4\%), PAP (92.6\%), P501s (95.1\%), NKX3.1 (91.4\%), and AMACR (77.3\%) (Fig. 1).

\section{Urothelial markers in the PAC group}

Only a small number of $\mathrm{PAC}$ was positive for urothelial markers. CK343E12, p63, thrombomodulin, S100P, and GATA3 immunostains were positive in $1.8 \%, 0 \%, 0 \%, 3.6 \%$, and $0 \%$ of PAC, respectively. The specificities of urothelial markers in PAC were as follows: CK34ßE12 (24.6\%), p63 (26.1\%), thrombomodulin (54.3\%), S100P (77.5\%), and GATA3 (15.2\%). The PPVs of urothelial markers in PAC were as follows: CK34 $\beta \mathrm{E} 12$ (1.9\%), p63 (0\%), thrombomodulin (0\%), S100P (11.4\%), and GATA3 (0\%). The NPVs of urothelial markers in PAC were as follows: CK34ßE12 (23.8\%), p63 (24.5\%), thrombomodulin (40.3\%), S100P (50.0\%), and GATA3 (15.9\%) (Fig. 1).

\section{Urothelial markers in the UC group}

The sensitivities of urothelial markers in the UC group were as follows: CK34ßE12 (75.4\%), p63 (73.9\%), thrombomodulin (45.7\%), S100P (22.5\%), and GATA3 (84.8\%). The specificities of urothelial markers in UC were as follows: CK34 $4 \mathrm{E} 12$ (98.2\%), p63 (100\%), thrombomodulin (100\%), S100P (96.4\%), and GATA3 (100\%). The PPVs of urothelial markers in UC were as follows: CK34ßE12 (98.1\%), p63 (100\%), thrombomodulin (100\%), S100P (88.6\%), and GATA3 (100\%). The NPVs of urothelial markers in UC were as follows: CK34 $3 \mathrm{E} 12$ (76.2\%), p63 (75.5\%), thrombomodulin (59.7\%), S100P (50.0\%), and GATA3 (84.1\%) (Figs. 2, 3).

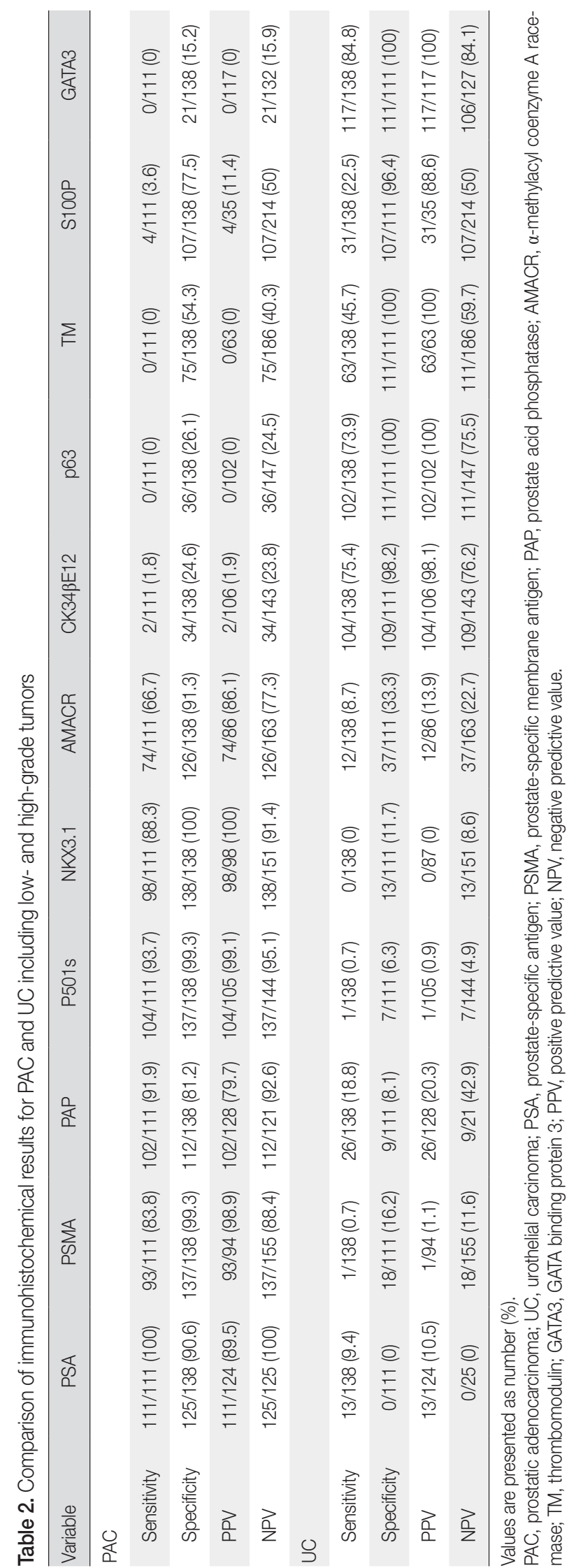



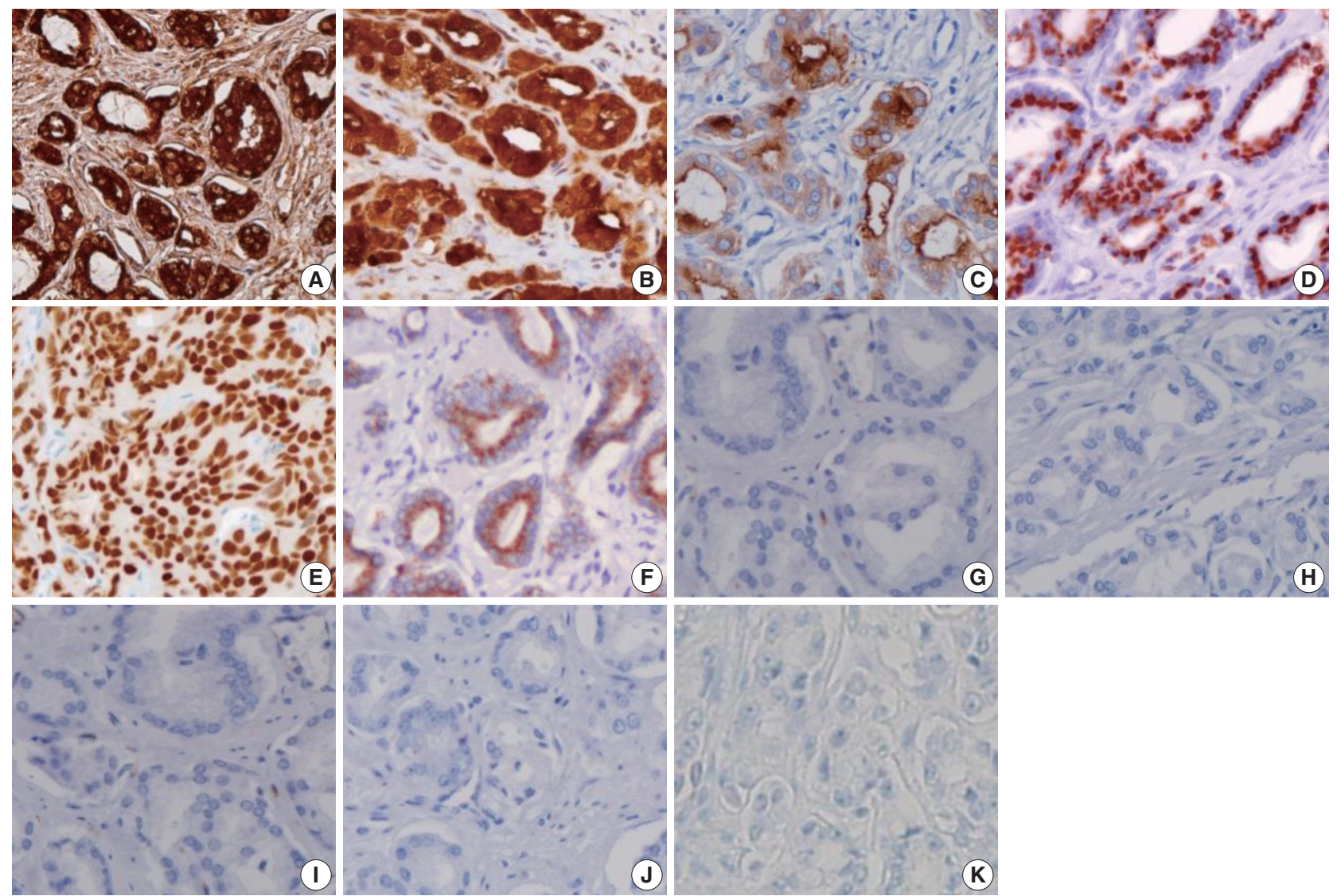

Fig. 1. Representative panel of immunohistochemical markers in most cases of prostate adenocarcinoma. Positive immunoreactivity for prostate-specific antigen (A), prostate-specific membrane antigen (B), prostate acid phosphatase (C), P501s (D), NKX3.1 (E), and $\alpha$-methylacyl coenzyme A racemase (F). Negative immunoreactivity for CK34ßE12 (G), p63 (H), thrombomodulin (I), S100P (J), and GATA binding protein $3(\mathrm{~K})$.

\section{Prostatic markers in the UC group}

The prostatic markers PSA, PSMA, PAP, P501s, NKX3.1, and AMACR were also positive in $9.4 \%, 0.7 \%, 18.8 \%, 0.7 \%$, $0 \%$, and $8.7 \%$ of UC, respectively. The specificities of prostatic markers in UC were as follows: PSA (0\%), PSMA (16.2\%), PAP (8.1\%), P501s (6.3\%), and NKX3.1 (11.7\%), and AMACR $(33.3 \%)$. The PPVs of prostatic markers in the UC group were as follows: PSA (10.5\%), PSMA (1.1\%), PAP (20.3\%), P501s (0.9\%), NKX3.1 (0\%), and AMACR (13.9\%). The NPVs of prostatic markers in UC were as follows: PSA (0\%), PSMA (11.6\%), PAP (42.9\%), P501s (4.9\%), NKX3.1 (8.6\%), and AMACR (22.7\%) (Figs. 2, 3).

\section{Subanalysis of high-grade PAC and high-grade UC}

We only included the patients with high-grade PAC ( 47 cases) and high-grade UC (110 cases) and analyzed the sensitivities and specificities of prostatic and urothelial markers. The results for high-grade PAC and high-grade UC were in the same range. No significant differences in the sensitivities and specificities were observed between the entire tumor groups and the highgrade groups (Table 3).

\section{DISCUSSION}

Although the pathologic identification of PAC and UC using hematoxylin and eosin staining is not difficult in most cases, some cases may present a challenging diagnosis because the histologic appearance of poorly differentiated PAC can be very similar to that of high-grade UC. ${ }^{3}$

High-grade PAC may have enlarged nuclei and prominent nucleoli similar to UC, but little variability in the nuclear size or shape is generally observed in PAC compared with UC., ${ }^{2,3}$ Additionally, even in high-grade PAC, there are few mitosis and pleomorphism compared with high-grade UC. ${ }^{3}$ Although highgrade UC commonly exhibits more pronounced pleomorphism compared with PAC, ${ }^{2,3}$ there have been cases of high-grade UC that were indistinguishable from high-grade PAC in terms of pleomorphism and cytologic atypia. ${ }^{3} \mathrm{UC}$ tends to grow in nests 

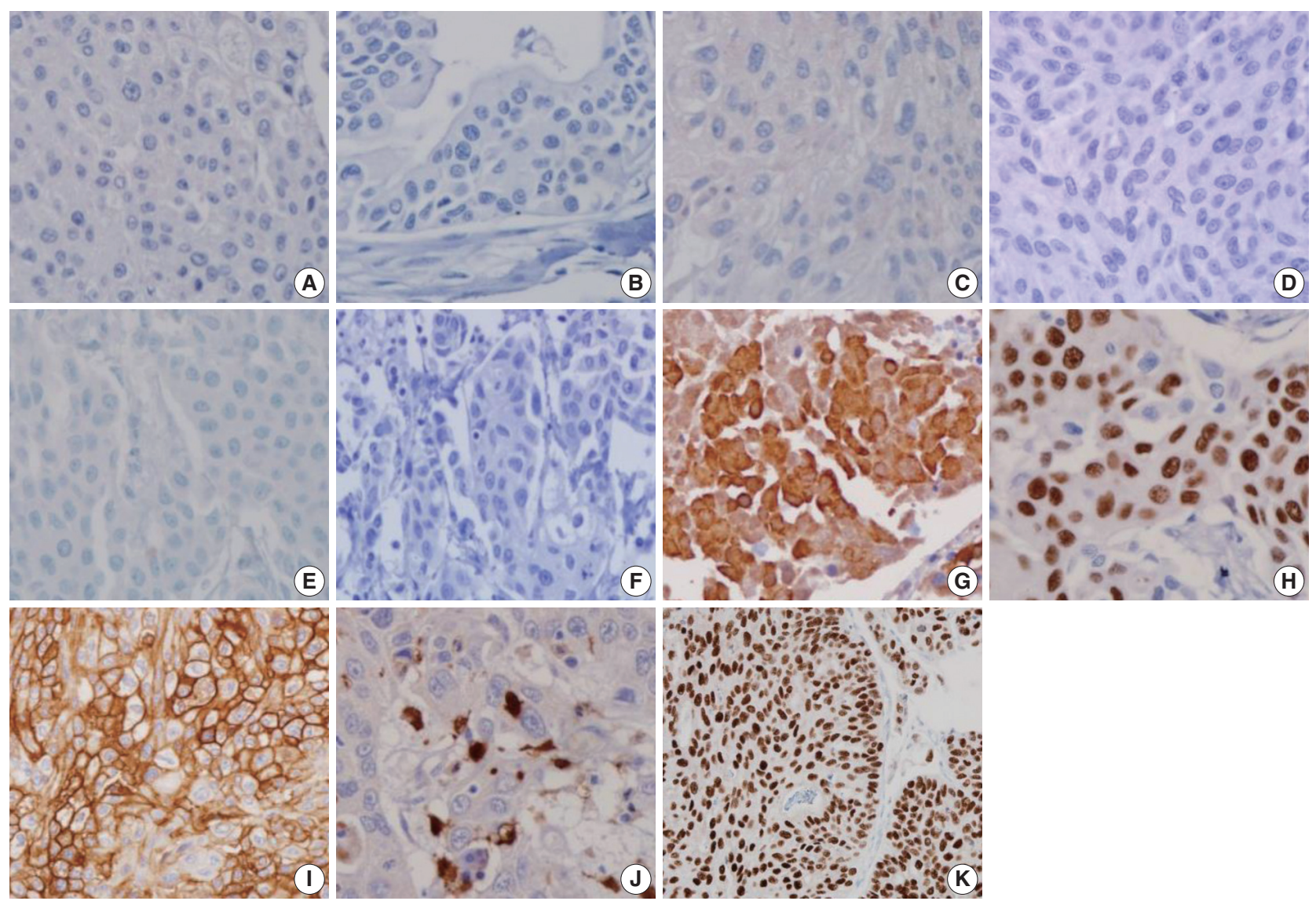

Fig. 2. Representative panel of immunohistochemical markers in most cases of urothelial adenocarcinoma. Negative immunoreactivity for prostate-specific antigen (A), prostate-specific membrane antigen (B), prostate acid phosphatase (C), P501s (D), NKX3.1 (E), and $\alpha$-methylacyl coenzyme A racemase (F). Positive immunoreactivity for CK34ßE12 (G), p63 (H), thrombomodulin (I), S100P (J), and GATA binding protein $3(\mathrm{~K})$.

and often shows conspicuous squamous differentiation and glassy eosinophilic cytoplasm. In contrast, the cytoplasm of PAC is generally pale and foamy. ${ }^{3}$ Additionally, the findings of focal cribriform glandular differentiation or infiltrating cords of cells are more typical features of PAC than UC., ${ }^{2,3}$ As the findings with routine hematoxylin and eosin staining may overlap, immunohistochemical staining may help solve the diagnostic dilemma. ${ }^{3}$ Particularly, in poorly differentiated carcinomas involving both the prostate and bladder without any glandular differentiation, the pathology of the case should be evaluated immunohistochemically (Table 4).

PSA, a serine protease member of the human glandular kallikrein family, is almost exclusively synthesized in the prostate ductal and acinar epithelium, making it a highly specific marker for the prostatic lineage. ${ }^{2}$ However, PSA has also been reported to be present in some non-prostatic tissue, such as the urethral, periurethral, and perianal glands. ${ }^{4}$ Extraprostatic neoplasms that frequently express PSA include urethral and periurethral ad- enocarcinoma, cloacogenic carcinoma, salivary gland pleomorphic adenoma, salivary duct carcinoma, and rare breast carcinomas. ${ }^{8}$ PSA has been shown to be a highly specific marker, but some authors suggest that there is an inverse correlation between the Gleason score and PSA staining intensity. Previous studies have reported that high-grade PAC that was completely negative for PSA stain ranged from $3 \%$ to $27 \% .^{3,5,6,9,10}$ However, in our study, no PAC specimens were devoid of PSA expression, including high-grade PAC, with $100 \%$ sensitivity. Therefore, PSA expression is very useful and valuable for clarifying the prostatic origin of tumors.

PSMA, a 750 amino acid type II membrane glycoprotein, is expressed by benign and malignant prostatic epithelial cells, with stronger staining observed in the latter. ${ }^{11}$ Although PSMA is a very specific marker of prostatic lineage, it is also expressed in non-prostatic tissues, such as the duodenal mucosa, neuroendocrine cells of colonic crypts, endothelial cells of some neoplasms, and proximal renal tubules. ${ }^{12,13}$ Some studies have re- 

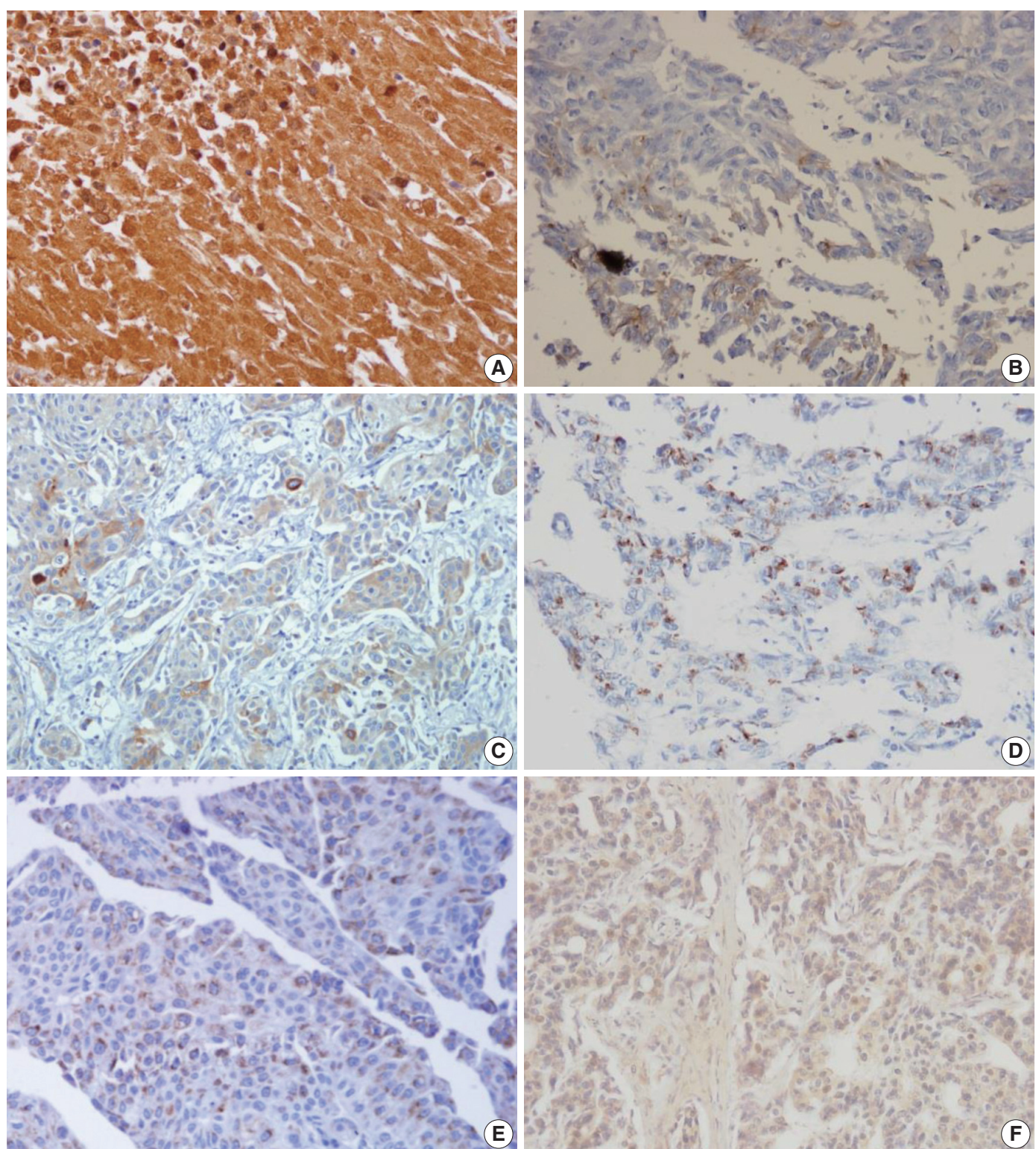

Fig. 3. (A) Prostate-specific antigen shows cytoplasmic staining of urothelial carcinoma. (B) Prostate-specific membrane antigen shows focal cytoplasmic staining of urothelial carcinoma. (C) Prostate acid phosphatase shows positivity in urothelial carcinoma. (D) P501S shows focal perinuclear cytoplasmic staining of urothelial carcinoma. (E) $\alpha$-Methylacyl coenzyme A racemase shows weakly positive staining of urothelial carcinoma. (F) S100P shows weak nuclear staining of prostate adenocarcinoma.

ported an inverse correlation between PSMA staining and the Gleason score. ${ }^{11,12}$ The sensitivities of PSMA for PAC ranged from $86.8 \%$ to $100 \%$ in various studies. ${ }^{3,12-15}$ In our study, the sensitivity of PSMA in PAC (83.8\%) was lower than PSA, but its specificity (99.3\%) was higher than PSA. PSMA has been reported to stain $11 \%$ of urinary bladder adenocarcinomas, a fact worth noting. ${ }^{16}$ We detected scattered patterns of positive PSMA staining in only one from 138 cases $(0.7 \%)$ of UC.

PAP is an early prostatic marker used to confirm the diagnosis of PAC5, ${ }^{15}$ and remains a specific marker for prostate tissue.
Mhawech et al. reported that $87 \%$ of high-grade PAC showed immunopositivity for PAP and observed an inverse correlation between the Gleason score and PAP staining. In this study, PAP was stained in $91.9 \%$ of PAC and showed a relative lack of specificity compared with PSMA ( $81.2 \%$ vs $99.3 \%)$, with a more variable staining pattern. Monoclonal antibodies to PAP have been reported to have lower sensitivities than their polyclonal counterparts but be more specific. ${ }^{2}$ PAP staining has been known to be consistently negative in $\mathrm{UC},{ }^{5,10,17}$ but a recent study reported immunopositivity in $11.1 \%$ of UC. ${ }^{6}$ Unexpectedly, we 
Table 3. Comparison of immunohistochemical results for high-grade PAC and high-grade UC

\begin{tabular}{|c|c|c|c|c|c|c|c|c|}
\hline Variable & PSA & PSMA & PAP & P501s & CK34ßE12 & p63 & S100P & GATA3 \\
\hline \multicolumn{9}{|l|}{$\overline{P A C}$} \\
\hline Sensitivity & $47 / 47(100)$ & 39/47 (83) & 43/47 (91.5) & $44 / 47$ (93.6) & $0 / 47(0)$ & $1 / 47(2.1)$ & $2 / 47(4.3)$ & $0 / 47(0)$ \\
\hline Specificity & $95 / 110$ (86.4) & $109 / 110(99.1)$ & $85 / 110(77.3)$ & $109 / 110(99.1)$ & $23 / 110(20.9)$ & $27 / 110(24.6)$ & $84 / 110(76.4)$ & $17 / 110(15.5)$ \\
\hline PPV & 47/62 (75.8) & $39 / 40(97.5)$ & 43/68 (63.2) & $44 / 45(98.8)$ & 0/87 (0) & $1 / 84(1.2)$ & $2 / 28(7.1)$ & 0/93 (0) \\
\hline NPV & $95 / 95(100)$ & $109 / 117$ (93.2) & 85/90 (95.5) & $109 / 112(97.3)$ & 23/70 (32.9) & $27 / 73(37)$ & $84 / 129(65.1)$ & 17/64 (26.6) \\
\hline \multicolumn{9}{|l|}{ UC } \\
\hline Sensitivity & 15/110 (13.6) & $1 / 110(0.9)$ & $25 / 110(22.7)$ & $1 / 110(0.9)$ & $87 / 110(79.1)$ & $83 / 110(75.5)$ & $26 / 110$ (23.6) & $93 / 110(84.5)$ \\
\hline Specificity & $0 / 47(0)$ & $8 / 47(17.0)$ & $4 / 47(8.5)$ & $3 / 47(6.4)$ & $47 / 47(100)$ & $46 / 47(97.9)$ & $45 / 47(95.7)$ & $47 / 47(100)$ \\
\hline PPV & $15 / 62(24.2)$ & $1 / 40(2.5)$ & 25/68 (36.8) & $1 / 45(2.2)$ & $87 / 87(100)$ & 83/84 (98.8) & $26 / 28$ (92.9) & $93 / 93(100)$ \\
\hline NPV & 0/95 (0) & $8 / 117(6.8)$ & $4 / 89(4.5)$ & $3 / 112(2.7)$ & $47 / 70(67.1)$ & $46 / 73(63)$ & $45 / 129(34.9)$ & $46 / 63(74.6)$ \\
\hline
\end{tabular}

Values are presented as number (\%).

PAC, prostatic adenocarcinoma; UC, urothelial carcinoma; PSA, prostate-specific antigen; PSMA, prostate-specific membrane antigen; PAP, prostate acid phosphatase; GATA3, GATA binding protein 3; PPV, positive predictive value; NPV, negative predictive value.

also detected PAP staining with a scattered pattern in 26 of 138 cases of UC (18.8\%).

P501s, a 553-amino acid protein located in the Golgi complex, is a newer prostate-specific protein identified by a combination of high-throughput microarray screening with cDNA subtraction. ${ }^{18} \mathrm{P} 501 \mathrm{~s}$ is expressed by benign and malignant prostatic epithelium and has not been detected in the urothelium or non-prostatic tissue. ${ }^{19}$ P501s was reported to be expressed in $94 \%$ of a total of observed 113 PAC cases, independent of the metastatic status and Gleason score. ${ }^{19}$ Chuang et al. ${ }^{3}$ reported that P501s was expressed in all 38 high-grade PAC cases. In the current study, P501s showed high sensitivity (93.7\%) and specificity (99.3\%) for PAC, and only one of 138 cases of UC $(0.7 \%)$ was positive for that marker. To date, P501s expression has not been shown in tumors except PAC, making it of great utility in differentiating poorly differentiated PAC from high-grade UC.,16

NKX3.1, a prostate specific androgen regulated homeobox gene ${ }^{20}$ is expressed in the prostatic epithelium, rare ureteral and urothelial cells, normal testis, lobular carcinoma of the breast, and bronchial mucous glands. ${ }^{21,22}$ Gelmann et al. ${ }^{22}$ reported that all 40 observed cases of UC were negative for NKX3.1. In the current study, none of the 138 cases of UC was positive for NKX3.1. The sensitivities of NKX3.1 for PAC reported in previous studies were $92.1 \%, 89.5 \%, 87.4 \%$, and $69.2 \%$. $321-23$ This study also showed a comparable result, with $88.3 \%$ sensitivity of NKX3.1 for PAC.

The AMACR, localized predominantly in peroxisomal structures, plays a critical role in peroxisomal beta oxidation of branched chain fatty acid. Jiang et al. ${ }^{24}$ demonstrated that both PAC and high-grade prostate intraepithelial neoplasia (HG-PIN) consistently revealed a significantly higher expression than normal epithelium. However, AMACR expression has repeatedly been demonstrated in HG-PIN and some benign mimickers of
PAC. Moreover, Kunju et al. ${ }^{6}$ reported that AMACR is expressed in $36 \%$ of UC cases. In our study, AMACR was expressed in $66.7 \%$ of 111 cases of PAC and $8.7 \%$ of 138 cases of UC. AMACR is less sensitive than other prostate markers for PAC and is of limited utility in resolving the difficult problems involving both the prostate and urinary bladder.

Although PSA, PSMA, PAP, P501s, and NKX3.1 are sensitive and specific markers for evaluating the prostatic origin of tumors, lack of staining was also detected for most markers, except PSA, in this study, at $16.2 \%$ for PSMA, $8.1 \%$ for PAP, $6.3 \%$ for P501s, and $11.7 \%$ for NKX3.1 of 111 PAC cases. Therefore, the lack of immunoreactivity of prostate markers in a poorly differentiated carcinoma does not exclude the possibility of a prostatic origin. In addition, false-positives were detected in UC in five of six established prostate markers in this study, ranging from $0.7 \%$ to $18.8 \%$, suggesting that the immunohistochemical panel is necessary and useful to discriminate poorly differentiated highgrade carcinomas involving both the prostate and bladder.

Many immunohistochemical stains have been investigated for UC, but no single marker has been found to be unequivocally diagnostic of urothelial origin. Thus, investigators have recommended a panel of markers to demonstrate the urothelial origin of tumor, such as CK34ßE12, p63, thrombomodulin, S100P, and GATA3.

The monoclonal antibody CK34 $3 \mathrm{E} 12$, which reactive specifically against high-molecular-weight cytokeratins (CKs), including CK1, CK5, CK14, and CK20, ${ }^{2}$ is an extremely sensitive marker of urothelial lineage. It is reported to match the sensitivity of p63 and surpass that of uroplakin III and thrombomodulin. ${ }^{3,25}$ Compared with previous studies showing sensitivities of $97.2 \%, 91.4 \%$, and $65.2 \%$ for CK34ßE12 in UC, $3,6,10$ our study found $75.4 \%$ sensitivity in UC. It is worth noting that $\mathrm{CK} 34 \beta \mathrm{E} 12$ can label squamous epithelia, including areas 


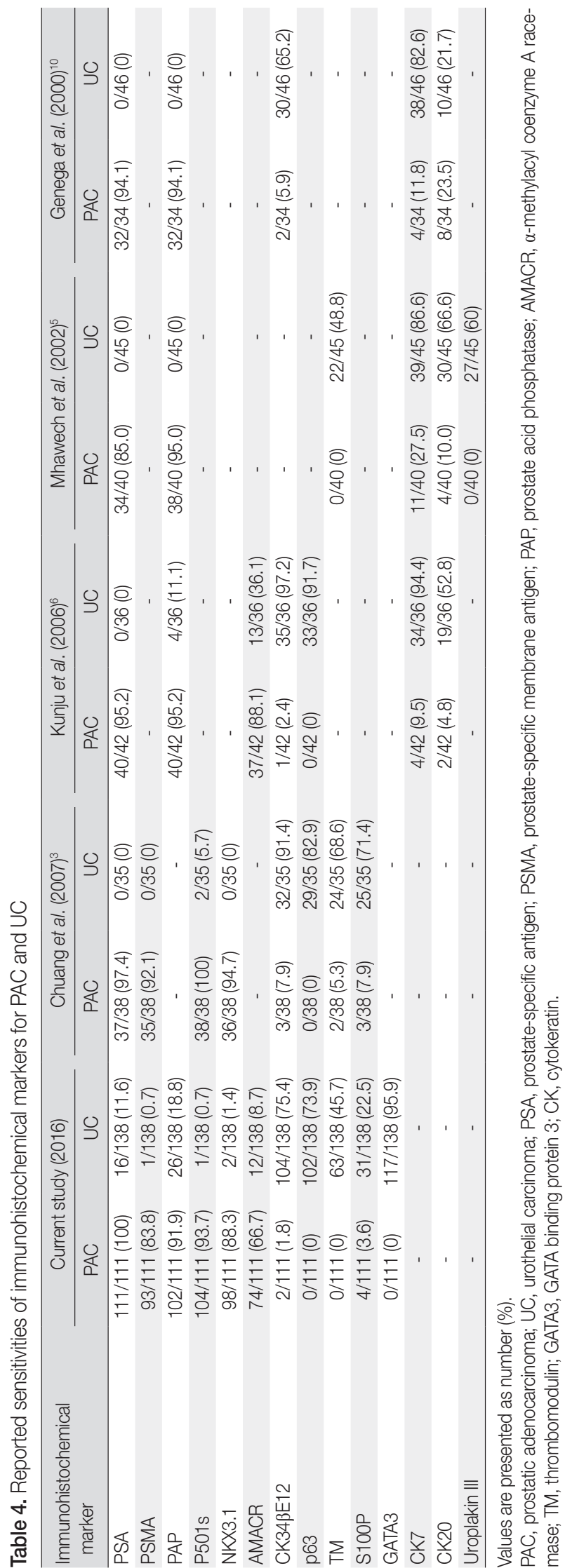

of squamous differentiation in recurrent PAC after therapy. Thus, Parwani $e t$ al. ${ }^{26}$ argued that immunopositivity for CK34 3 E12 restricted to areas of squamous differentiation does not exclude the possibility of PAC.

$\mathrm{p} 63$, a homologue of the $\mathrm{p} 53$ tumor suppressor gene, encodes at least six different proteins with a wide range of biologic functions, including a role in urothelial differentiation. ${ }^{2}$ Immunostaining for $\mathrm{p} 63$ is typically present in more than $90 \%$ of the nuclei of the normal urothelia. ${ }^{2}$ Many UCs retain a pattern of p63 expression, but p63 expression may be partially lost in highgrade UC. ${ }^{3,27}$ Although p63 sensitivity for UC in our study $(73.9 \%)$ was lower than that of previous studies $(82.9 \%-$ $91.7 \%),{ }^{3,6}$ its specificity was $100 \%$ for UC.

Thrombomodulin, also designated CD141, is an endothelial cell associated cofactor for the thrombin-mediated activator of protein C. ${ }^{2}$ Previous studies have shown that thrombomodulin was immunostained in $48.8 \%-68.6 \%$ of UC, 3,5 but our study found a slightly lower expression at $45.7 \%$.

$\mathrm{S} 100 \mathrm{P}$ is highly expressed in the urothelial epithelium. ${ }^{28} \mathrm{Hig}$ gins et al ${ }^{28}$ reported that the polyclonal antibody against $\mathrm{S100P}$ labeled $85 \%$ of UC and $3 \%$ of PAC, whereas the monoclonal antibody against S100P detected $77 \%$ of UC and 2\% of PAC. Chuang et $a l^{3}$ also reported that the monoclonal S100P detected $51.4 \%$ of UC and $7.9 \%$ of PAC. In our study with a monoclonal antibody against S100P, $22.5 \%$ of UC and $3.6 \%$ of PAC were stained, which was less than in previous studies. ${ }^{3,28}$

Although CK34ßE12 and p63 have been reported to intermittently label PAC in a non-basal cell distribution, thrombomodulin has not been reported to show cross reactivity. ${ }^{5,6,10} \mathrm{We}$ found that CK34ßE12 and p63 immunostains were superior to

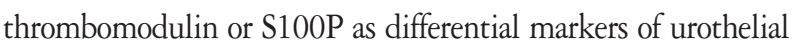
origin. Only a few scattered cells of PAC were labeled with CK34ßE12 (1.8\%) and S100P (3.6\%), but no PAC was immunopositive for thrombomodulin or p63 in our study.

GATA3 is a member of a zinc finger transcription factor family that plays an important role in promoting and directing cell proliferation, differentiation, and development. ${ }^{2}$ GATA3 is a very sensitive marker for $\mathrm{UC}$, and it is also highly specific in excluding high-grade PAC. ${ }^{29}$ Chang et al. ${ }^{29}$ reported that none of the 38 high-grade PACs was positive for GATA3. In this study, the sensitivity of GATA3 was $0 \%$ in PAC and $84.8 \%$ in UC. Uroplakin III is considered the most specific marker for urothelial differentiation, but it has not received popularity due to the lack of uniform expression in UCs. ${ }^{29}$ Our study has some limitations because we did not include studies of Uroplakin III.

In conclusion, prostatic markers, including PSA, PSMA, PAP, 
and P501s, are very useful for distinguishing PAC from UC. Urothelial markers are less sensitive in identifying UC but rarely stain PAC. In the current study, we found that PSA is most sensitive prostatic marker for distinguishing PAC from UC cases with high sensitivity and negative predictive value. In addition, NKX3.1 is the most specific prostatic marker for distinguishing PAC from UC cases with high specificity and positive predictive value. p63 and thrombomodulin are the most specific urothelial markers for distinguishing UC from PAC cases with high specificities. GATA3 was positive in 117 of 137 cases of UCs and none of the 111 PACs was positive for GATA3. We found that the best combination of immunohistochemical markers for distinguishing PAC from UC is panels consisting of PSA, NKX3.1, p63, thrombomodulin, and GATA3. The optimal combination of immunohistochemical panels of prostatic and urothelial markers could improve the ability to establish the pathologic diagnosis of poorly differentiated high-grade carcinomas involving either the prostate or urinary bladder.

\section{Conflicts of Interest}

No potential conflict of interest relevant to this article was reported.

\section{REFERENCES}

1. Bates AW, Baithun SI. Secondary neoplasms of the bladder are histological mimics of nontransitional cell primary tumours: clinicopathological and histological features of 282 cases. Histopathology 2000; 36: 32-40.

2. Dabbs DJ. Diagnostic imunohistochemistry. 3rd ed. Philadelphia: Saunders-Elsevier, 2010; 621-5.

3. Chuang AY, DeMarzo AM, Veltri RW, Sharma RB, Bieberich CJ, Epstein JI. Immunohistochemical differentiation of high-grade prostate carcinoma from urothelial carcinoma. Am J Surg Pathol 2007; 31: 1246-55.

4. Varma M, Jasani B. Diagnostic utility of immunohistochemistry in morphologically difficult prostate cancer: review of current literature. Histopathology 2005; 47: 1-16.

5. Mhawech P, Uchida T, Pelte MF. Immunohistochemical profile of high-grade urothelial bladder carcinoma and prostate adenocarcinoma. Hum Pathol 2002; 33: 1136-40.

6. Kunju LP, Mehra R, Snyder M, Shah RB. Prostate-specific antigen, high-molecular-weight cytokeratin (clone 34betaE12), and/or p63: an optimal immunohistochemical panel to distinguish poorly differentiated prostate adenocarcinoma from urothelial carcinoma.
Am J Clin Pathol 2006; 125: 675-81.

7. Chibber PJ, McIntyre MA, Hindmarsh JR, Hargreave TB, Newsam JE, Chisholm GD. Transitional cell carcinoma involving the prostate. Br J Urol 1981; 53: 605-9.

8. Kamoshida S, Tsutsumi Y. Extraprostatic localization of prostatic acid phosphatase and prostate-specific antigen: distribution in cloacogenic glandular epithelium and sex-dependent expression in human anal gland. Hum Pathol 1990; 21: 1108-11.

9. Goldstein NS. Immunophenotypic characterization of 225 prostate adenocarcinomas with intermediate or high Gleason scores. Am J Clin Pathol 2002; 117: 471-7.

10. Genega EM, Hutchinson B, Reuter VE, Gaudin PB. Immunophenotype of high-grade prostatic adenocarcinoma and urothelial carcinoma. Mod Pathol 2000; 13: 1186-91.

11. Marchal C, Redondo M, Padilla M, et al. Expression of prostate specific membrane antigen (PSMA) in prostatic adenocarcinoma and prostatic intraepithelial neoplasia. Histol Histopathol 2004; 19: 715-8.

12. Bostwick DG, Pacelli A, Blute M, Roche P, Murphy GP. Prostate specific membrane antigen expression in prostatic intraepithelial neoplasia and adenocarcinoma: a study of 184 cases. Cancer 1998; 82: 2256-61.

13. Silver DA, Pellicer I, Fair WR, Heston WD, Cordon-Cardo C. Prostate-specific membrane antigen expression in normal and malignant human tissues. Clin Cancer Res 1997; 3: 81-5.

14. Chang SS, Reuter VE, Heston WD, Gaudin PB. Comparison of anti-prostate-specific membrane antigen antibodies and other immunomarkers in metastatic prostate carcinoma. Urology 2001; 57: 1179-83.

15. Sweat SD, Pacelli A, Murphy GP, Bostwick DG. Prostate-specific membrane antigen expression is greatest in prostate adenocarcinoma and lymph node metastases. Urology 1998; 52: 637-40.

16. Lane Z, Hansel DE, Epstein JI. Immunohistochemical expression of prostatic antigens in adenocarcinoma and villous adenoma of the urinary bladder. Am J Surg Pathol 2008; 32: 1322-6.

17. Bassily NH, Vallorosi CJ, Akdas G, Montie JE, Rubin MA. Coordinate expression of cytokeratins 7 and 20 in prostate adenocarcinoma and bladder urothelial carcinoma. Am J Clin Pathol 2000; 113: 383-8.

18. Xu J, Kalos M, Stolk JA, et al. Identification and characterization of prostein, a novel prostate-specific protein. Cancer Res 2001; 61: 1563-8.

19. Kalos M, Askaa J, Hylander BL, et al. Prostein expression is highly restricted to normal and malignant prostate tissues. Prostate 2004; 60: 246-56.

20. He WW, Sciavolino PJ, Wing J, et al. A novel human prostate-spe- 
cific, androgen-regulated homeobox gene (NKX3.1) that maps to 8 p21, a region frequently deleted in prostate cancer. Genomics 1997; 43: 69-77.

21. Bowen C, Bubendorf L, Voeller HJ, et al. Loss of NKX3.1 expression in human prostate cancers correlates with tumor progression. Cancer Res 2000; 60: 6111-5.

22. Gelmann EP, Bowen C, Bubendorf L. Expression of NKX3.1 in normal and malignant tissues. Prostate 2003; 55: 111-7.

23. Aslan G, Irer B, Tuna B, Yorukoglu K, Saatcioglu F, Celebi I. Analysis of NKX3.1 expression in prostate cancer tissues and correlation with clinicopathologic features. Pathol Res Pract 2006; 202: 93-8.

24. Jiang Z, Li C, Fischer A, Dresser K, Woda BA. Using an AMACR (P504S)/34betaE12/p63 cocktail for the detection of small focal prostate carcinoma in needle biopsy specimens. Am J Clin Pathol 2005; 123: 231-6.

25. Parker DC, Folpe AL, Bell J, et al. Potential utility of uroplakin III, thrombomodulin, high molecular weight cytokeratin, and cytoker- atin 20 in noninvasive, invasive, and metastatic urothelial (transitional cell) carcinomas. Am J Surg Pathol 2003; 27: 1-10.

26. Parwani AV, Kronz JD, Genega EM, Gaudin P, Chang S, Epstein JI. Prostate carcinoma with squamous differentiation: an analysis of 33 cases. Am J Surg Pathol 2004; 28: 651-7.

27. Comperat E, Camparo P, Haus R, et al. Immunohistochemical expression of p63, p53 and MIB-1 in urinary bladder carcinoma: a tissue microarray study of 158 cases. Virchows Arch 2006; 448: 319-24.

28. Higgins JP, Kaygusuz G, Wang L, et al. Placental S100 (S100P) and GATA3: markers for transitional epithelium and urothelial carcinoma discovered by complementary DNA microarray. Am J Surg Pathol 2007; 31: 673-80.

29. Chang A, Amin A, Gabrielson E, et al. Utility of GATA3 immunohistochemistry in differentiating urothelial carcinoma from prostate adenocarcinoma and squamous cell carcinomas of the uterine cervix, anus, and lung. Am J Surg Pathol 2012; 36: 1472-6. 